\title{
SINGULARITIES OF ENERGY-MINIMIZING MAPS FROM THE BALL TO THE SPHERE
}

\author{
FREDERICK J. ALMGREN, JR. AND ELLIOTT H. LIEB
}

We study maps $\varphi$ from the unit ball $\mathbf{B}$ in $\mathbf{R}^{3}$ to the unit sphere $\mathbf{S}^{2}$ in $\mathbf{R}^{3}$ which minimize Dirichlet's energy integral

$$
\mathcal{E}(\varphi)=\int_{\mathbf{B}}|\nabla \varphi|^{2} d V
$$

If such a $\varphi$ minimized Dirichlet's integral among mappings into $\mathbf{R}^{3}$ rather than being constrained to lie in $\mathbf{S}^{2}$ it would then be a classical smooth harmonic function. A minimizing constrained $\varphi$, however, sometimes has isolated point discontinuities. We here announce several new estimates on the number and arrangement of such singular points $[\mathbf{A L}]$. The $\varphi$ 's we consider have well defined values $\psi$ on the boundary $\partial \mathbf{B}$ of $\mathbf{B}$, and the boundary Dirichlet's energy integral is

$$
\partial \mathcal{E}(\psi)=\int_{\partial \mathbf{B}}\left|\nabla_{T} \psi\right|^{2} d A
$$

where $\nabla_{T} \psi$ denotes the tangential gradient. In our theorems and examples below each $\psi$ has finite energy. One of our principal results is

MAIN THEOREM. Suppose $\varphi$ minimizes Dirichlet's integral among all functions mapping $\mathbf{B}$ to $\mathbf{S}^{2}$ and having boundary value function $\psi$ on $\partial \mathbf{B}$. Then the number of points of discontinuity of $\varphi$ is bounded by a constant times $\partial \mathcal{E}(\psi)$.

This linear law is noteworthy because examples illustrate linear growth of the number of singularities with $\partial \mathcal{E}(\psi)$ while other examples show that the number of singularities cannot be bounded by $\mathcal{E}(\varphi)$. This shows that the number and location of singularities cannot be inferred from simple energy comparisons alone. The subtlety of this estimate is further illustrated by

EXAMPLES. There are boundary value functions $\psi$ for which the minimizing $\varphi$ 's are unique and have an arbitrarily large number of singular points stacked arbitrarily high near the boundary-like bubbles in a pan of water that is almost ready to boil. The number of stacks is also arbitrarily large.

Such examples show the necessity of an analysis containing several different length scales in proving the principal result above--the length scale of a singular point is its distance to the boundary.

Received by the editors April 20, 1987.

1980 Mathematics Subject Classification(1985 Revision). Primary 58E20; Secondary 58E30, $82 \mathrm{~A} 50$. 
One might expect that if $\psi$ mapped $\partial \mathbf{B}$ to cover only small area in $\mathbf{S}^{2}$ then there could not be too many singular points of $\varphi$ in $\mathbf{B}$. Indeed, prior to our work, all examples of boundary values $\psi$ with many singularities also had boundary mapping area proportional to $\partial \mathcal{E}(\psi)$. Such a relationship turns out not to hold in general and, as another of our principal results, we show

EXAMPLES. For any preassigned number $N$, there is a smooth boundary value mapping $\psi$ of $\partial \mathbf{B}$ to $\mathbf{S}^{2}$ with the following properties: (i) the image of $\psi$ in $\mathbf{S}^{2}$ consists of a single smooth curve $\Gamma$ near the equator ( $\psi$ thus has zero mapping area), and (ii) any minimizing $\varphi$ has at least $N$ singularities.

One key ingredient of these examples is the existence of two different parametrizations of $\Gamma$ from the boundary $\partial D$ of the unit disk $D$ such that the least energy extension of the first parametrization maps $D$ to cover the north pole of $\mathbf{S}^{2}$ while the least energy extension of the second parametrization maps $D$ to cover the south pole. This then leads directly to an example in which B is replaced by a large solid torus with cross-section $D$ and the boundary parametrizations alternate as one goes along the torus. We effectively embed such a torus in $\mathbf{B}$ using the conformal equivalence between the disk and the half-plane.

Another natural question one might ask is whether minimizers respect boundary value symmetries (if any), as is true for classical harmonic functions. This is not the case as we illustrate by

EXAMPLES. There are boundary value functions $\psi$ which are symmetric about the midplane of $\mathbf{B}$ but for which any minimizer cannot possess such a symmetry (nor can its set of discontinuities).

The basic existence and regularity (interior and boundary) theorems for $\varphi$ 's and $\psi$ 's as above appear in papers of R. Schoen and K. Uhlenbeck [SU1, SU2]. It is their work which guarantees that the interior discontinuities for $\varphi$ 's are isolated. The uniqueness of tangential approximations at such points of discontinuity follows from the work of L. Simon [S]. Following initial estimates by R. Hardt, D. Kinderlehrer, and M. Luskin [HKL], H. Brezis, J.-M. Coron, and Lieb showed that the only possible tangential approximation to a minimizing $\varphi$ at any singular point is the function $x /|x|$ composed with an orthogonal mapping of $\mathbf{S}^{2}$ [BCL]. Hardt and F. H. Lin showed in [HL] how to construct boundary values $\psi$ which would guarantee many singularities in a minimizing $\varphi$. Except for this, little was known about the number and location of singularities in a minimizer when the present work began.

Much of the basic analysis in the literature mentioned above has been based ultimately on compactness arguments, i.e. failure of a desired estimate for all constants leads to an impossible situation. Such compactness arguments are central to the present work as well; they lead fairly directly to the following important estimate (Hardt and Lin have informed us of their independent discovery of this fact).

THEOREM. The distance between any two singularities $p$ and $q$ in a minimizing $\varphi$ is at least a fixed constant multiple of the distance from $p$ to $\partial \mathbf{B}$. 
Another compactness argument which combines the theorem above with the boundary regularity theory enables us to conclude that the existence of a singularity at distance $\delta$ from $\partial \mathbf{B}$ implies that the boundary function $\psi$ must have nearby Dirichlet integral at scales comparable to $\delta$ independent of boundary energy distribution at much larger or much smaller scales. A combinatorial analysis on a Cayley tree based on these differing length scales permits us to sum these different energies in proving our main theorem.

As one might suspect our main theorem remains true (with appropriate constants) if $\mathbf{B}$ is replaced by considerably more general domains in $\mathbf{R}^{3}$, while the second theorem holds with the same constant.

One of the original motivations for studying mappings to $\mathbf{S}^{2}$ (or to $\mathbf{R} \mathbf{P}^{2}$ ) was the mathematical analysis of liquid crystal configurations--in this context one usually regards $\varphi$ as a unit vectorfield in $\mathbf{B}$. Because we base our analysis on compactness arguments we can also readily conclude that a unit vectorfield $\varphi$ which minimizes any nematic liquid crystal energy integral sufficiently close to Dirichlet's integral must have at most isolated point discontinuities and the number of these discontinuities is dominated by boundary energy.

\section{REFERENCES}

[AL] F. J. Almgren, Jr. and E. H. Lieb, Singularities of energy-minimizing maps from the ball to the sphere: examples, counterexamples, and bounds, in preparation.

[BCL] H. Brezis, J.-M. Coron and E. H. Lieb, Harmonic maps with defects, Comm. Math. Physics 107 (1986), 649-705.

[HKL] R. Hardt, D. Kinderlehrer and M. Luskin, Remarks about the mathematical theory of liquid crystals, IMA Preprint \#276, October 1986. $1-10$.

[HL] R. Hardt and F. H. Lin, A remark on $\mathbf{H}^{1}$ mappings, Manuscripta Math. 56 (1986),

[SU1] R. Schoen and K. Uhlenbeck, A regularity theory for harmonic maps, J. Differential Geom. 17 (1982), 307-335.

[SU2] _ Boundary regularity and the Dirichlet problem of harmonic maps, J. Differential Geom. 18 (1983), 253-268.

[S] L. Simon, Asymptotics for a class of non-linear evolution equations with applications to geometric problems, Ann. of Math. (2) 118 (1983), 525-571.

Department of Mathematics, Princeton University, Princeton, NeW JERSEY 08544 